Article

\title{
Flame Retardant Multilayered Coatings on Acrylic Fabrics Prepared by One-Step Deposition of Chitosan/Montmorillonite Complexes
}

\author{
Federico Carosio ${ }^{1, *}$ and Jenny Alongi 2 (iD \\ 1 Dipartimento di Scienza Applicata e Tecnologia, Politecnico di Torino, Alessandria Campus, \\ Viale Teresa Michel 5, 15121 Alessandria, Italy \\ 2 Dipartimento di Chimica, Università degli Studi di Milano, Via C. Golgi 19, 20133 Milano, Italy; \\ jenny.alongi@unimi.it \\ * Correspondence: federico.carosio@polito.it; Tel.: +39-0131-229303
}

Received: 26 April 2018; Accepted: 16 May 2018; Published:4 June 2018

\begin{abstract}
Multilayered coatings deposited using the layer-by-layer (LbL) assembly technique have attracted great interest in recent years as a sustainable and efficient solution for conferring flame retardant properties to fabrics. The unique structure and interaction established upon the coating assembly are the key factors for successful flame retardant properties. In this study we aimed at the deposition of multilayered coatings comprising chitosan and montmorillonite with a LbL-like structure and interactions by the simple processing of compacted chitosan/montmorillonite complexes obtained by the direct mixing of an oppositely charged solution/suspension. Upon drying, the prepared complex yielded a continuous coating characterized by a brick-and-mortar multi-layered structure, in which oriented clay nanoplatelets were held together by a continuous chitosan matrix. When deposited on acrylic fabrics these coatings were able to suppress the melt-dripping phenomenon, and at 10 and $20 \%$ add-ons achieved self-extinguishing behavior within a few seconds after ignition. Cone calorimetry testing revealed an increase in time to ignition (up to $+46 \%$ ) and considerable reductions of the rates at which heat is released (up to -62 and $-49 \%$ for peak of heat release rate and total heat release, respectively). A reduction in the total smoke release (up to $-49 \%$ ) was also observed.
\end{abstract}

Keywords: acrylic fibers; chitosan; montmorillonite; flame retardancy; cone calorimetry

\section{Introduction}

In recent years, synthetic fibers surpassed the production of natural fibers and have become leading materials in the textile industry. Among synthetic products, acrylic fibers are among the most important man-made fibers after polyesters and nylons [1]. Similarly to other synthetic fibers, acrylic fibers can easily catch fire and quickly burn, thus representing a potential risk for the ignition and spreading of fire [2]. For this reason, both academic and industrial scientists have devoted a lot of effort to developing practical and efficient flame retardant (FR) solutions capable of improving the ignitability of acrylic fibers [3-6]. Within this context, the phosphorus-containing comonomers developed in the 1950-60s mostly resulted in the best FR properties with respect to the use of other additives. In addition, over the years, different spinning dope additives (e.g., antimony and tin esters or oxides, silica, halogenated and/or phosphorus compounds) have been also investigated [1,7]. Char-forming FR compounds have proven to be among the most effective FR solutions with respect to vapor-phase-inhibiting FRs [1].

Recently, another strategy based on the surface deposition of FR nanostructured coatings has been developed for fabrics and other substrates [8]. In particular, the layer-by-layer (LbL) 
deposition technique has proven to be capable of delivering exceptional FR properties while relying on relatively simple and green processing conditions $[9,10]$. Indeed, LbL allows for the construction of nanostructured coatings on the basis of the sequential adsorption on the selected substrate of oppositely charged polyelectrolytes or nanoparticles from diluted (0.1-1 wt. \%) aqueous solutions/suspensions [11,12]. The vast variety of available reagents has allowed for the deposition of FR coatings acting with different flame retardant mechanisms [8]. For instance, thermally shielding coatings can be deposited by combining lamellar or spherical nanoparticles, such as sodium montmorillonite, vermiculite or silica [13-16]. Similarly, coatings characterized by char forming/intumescent features can be achieved by employing polyphosphates, such as ammonium polyphosphate, sodium hexametaphosphate in combination with synthetic or natural polyelectrolytes such as polyallylamine, chitosan and starch [17-20]. In addition, by controlling the deposition parameters such as molecular weight, ionic strength and solution/suspension concentration, it is possible to change the resulting FR properties [21,22].

One of the key features that has made LbL coatings so successful is the unique structure and intimate interactions that are established during the polyelectrolyte/polyelectrolyte or polyelectrolyte/nanoparticles complexation reaction that is at the base of LbL coating assembly [12,23]. The formation of such complexes (PECs) allows for synergistic rather than simply additive interactions between the coating constituents, which in turn provide exceptional FR properties [24,25].

However, the main drawback of LbL is the excessive amount of time and deposition steps required to achieve the desired FR properties. In order to improve the efficiency of the process, recent research has been focused on the production of metastable or stable PECs in solution aiming to achieve the deposition of a LbL-like coating with few deposition steps [26,27]. Stable PEC solutions can be obtained by mixing two oppositely charged solutions/suspensions under precise $w t / w t$ ratios or high ionic strength conditions [28]. This technique has been used to prepare PECs made of branched polyethyleneimine and polyacrylic acid that were subsequently doctor-bladed on the surface of a polyester film in order to deposit a gas barrier coating [29]. Another approach involves the preparation of PECs followed by their compaction in gels of different density and viscosity by exploiting centrifugal forces [28].

In this manuscript, the production of chitosan $(\mathrm{CH})$ and sodium montmorillonite (MMT) complexes and their use as an FR LbL-mimicking solution for acrylic fibers is proposed. $\mathrm{CH}$ solutions and MMT suspensions at different concentrations were mixed and the produced PECs suspension was compacted by centrifugal forces. The resulting gel was then deposited on acrylic fabrics using a simple doctor-blade coating technique in order to obtain a FR finishing characterized by a LbL-like structure and interactions, as schematized in Figure 1, which depicts the approach adopted in this work.

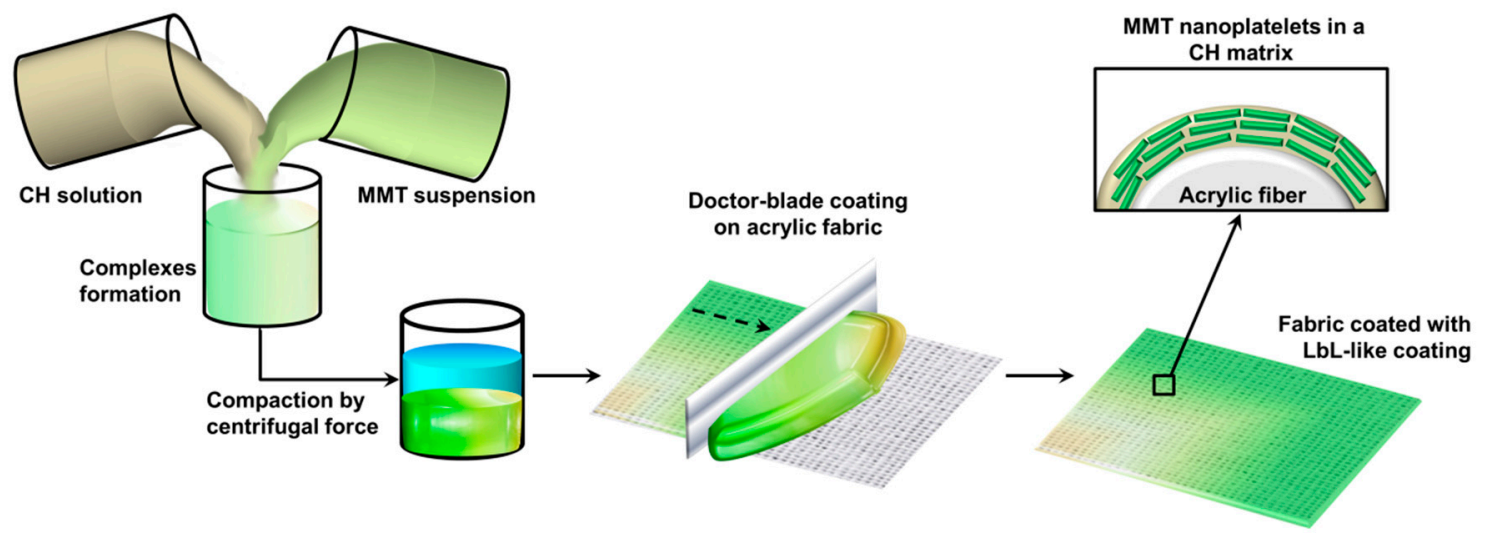

Figure 1. Schematic description of the process adopted to coat acrylic fabric: chitosan $(\mathrm{CH})$ solution and montmorillonite (MMT) suspension are mixed in controlled conditions and then centrifuged, the resulting gel-like precipitate is then doctor-bladed on the fabrics. 
The aim is to deposit a coating characterized by the so-defined "brick and mortar" structure, where MMT nanoplatelets are embedded within a continuous $\mathrm{CH}$ matrix and interact through electrostatic interactions [16]. MMT is preferentially oriented parallel to the surface; it is expected that, during combustion, the charred residue of the $\mathrm{CH}$ matrix holds together the nanoplatelets and allows for the build-up of a barrier that may block or limit heat, oxygen and mass transfer towards the substrate and volatile release to feed the flame with a subsequent FR effect [30].

The morphological analysis and chemical characterizations were carried out by scanning electron microscopy and infrared spectroscopy, respectively. The combustion properties were evaluated in terms of their resistance to the application of a flame (flammability in horizontal configuration) and resistance to the exposure of an incident heat flux typical of a fire in the early stage (cone calorimetry test).

\section{Materials and Methods}

\subsection{Materials}

The acrylic fabric (copolymer: $90 \%$ of polyacrylonitrile and 10\% methyl acrylate) hereafter coded as A with a $400 \mathrm{~g} / \mathrm{m}^{2}$ grammage was purchased from Fratelli Ballesio s.r.l. (Torino, Italy). The fabric was washed in deionized water and subsequently dried before testing/coating. Chitosan (low molecular weight, $<20,000 \mathrm{Da}, 75-85 \%$ deacetylated) and citric acid were purchased from Sigma Aldrich (Milwaukee, WI, USA) and used as received. Chitosan was dissolved in a $0.1 \mathrm{M}$ citric acid solution in order to prepare a $0.1 \mathrm{wt}$. \% solution. The sodium montmorillonite was purchased from Southern clays (Gonzales, TX, USA). The MMT was used as received to prepare $2 \mathrm{wt}$. \% water suspensions that were kept under magnetic stirring for $24 \mathrm{~h}$. Prior to use, the MMT suspensions were centrifuged ( $5 \mathrm{~min} @ 4400 \mathrm{rpm}$ ) in order to remove big aggregates; after this procedure the suspension had a final concentration of $1.5 \pm 0.1 \mathrm{wt} . \%$. $\mathrm{CH}$ solutions and MMT suspensions were prepared using 18.2 $\mathrm{M} \Omega$ deionized water supplied by a Q20 Millipore system (Milano, Italy).

\subsection{Compacted Complexes: Preparation and Deposition on Fabrics}

Initially, $\mathrm{CH}$ solution and MMT suspension were mixed $(1: 1 v / v)$ and kept under magnetic stirring for $20 \mathrm{~min}$, yielding a pearlescent greenish suspension. Then, the so-prepared mixture was centrifuged for $5 \mathrm{~min}$ at $4400 \mathrm{rpm}$, the supernatant was subsequently removed and the precipitate (gel) collected and stored in a fridge at $4{ }^{\circ} \mathrm{C}$.

Before the coating procedure, fabrics were cut in $100 \times 100 \mathrm{~mm}^{2}$ pieces and weighted. Then, the prepared gel was deposited on the surface of the fabric using a doctor-blading procedure (Figure 1); the treated fabric was then dried in a ventilated oven at $80^{\circ} \mathrm{C}$. These steps were repeated on both sides of the fabric, each time targeting a wet pick up of $200 \mathrm{wt}$. \%. The process was repeated until a total coating add-on of 5,10 and $20 \pm 0.5 \%$ was achieved.

\subsection{Characterization Techniques}

The cross section morphology of the $\mathrm{CH} / \mathrm{MMT}$ dried complexes samples was studied using a ZEISS, FEG model MERLIN field emission scanning electron microscopy (FESEM) (beam voltage: $5 \mathrm{kV}$ ). The cross-section obtained by fragile fracture was mounted on the FESEM stubs, fixed with aluminum tape and chromium metalized prior to observation.

The surface morphology of untreated and treated fabrics was investigated using a LEO-1450VP scanning electron microscope (beam voltage: $5 \mathrm{kV})$. Small $\left(10 \times 10 \mathrm{~mm}^{2}\right)$ samples mounted on conductive tape were gold coated prior to SEM observation.

Attenuated total reflectance (ATR) spectroscopy was used to evaluate the changes in surface chemistry after the coating procedure. ATR spectra were recorded at room temperature in the range $4000-700 \mathrm{~cm}^{-1}$ (16 scans and $4 \mathrm{~cm}^{-1}$ resolution), using a Frontier FT-IR/FIR spectrophotometer, equipped with a diamond crystal (penetration depth of $1.66 \mu \mathrm{m}$ ). 
Flammability tests were carried out in horizontal configuration on rectangular specimens $\left(50 \times 100 \mathrm{~mm}^{2}\right)$. Prepared samples were blocked in a frame with a 45 degrees inclination along the horizontal direction. Then, a blue methane flame ( $20 \mathrm{~mm}$ length) was applied on the short site of the specimen for $6 \mathrm{~s}$. These tests were repeated three times for each formulation. During the test the flame propagation rate, the formation of incandescent melt droplets, ignition of dry cotton placed underneath the testing specimen and final residue were evaluated.

Forced combustion tests were performed using cone calorimetry (fire testing technology) on square samples $\left(100 \times 100 \mathrm{~mm}^{2}\right)$ in the horizontal configuration. The measurements were carried out under a $25 \mathrm{~kW} / \mathrm{m}^{2}$ irradiative heat flux, following a previously described procedure [17]. Parameters such as time to ignition (TTI, s), peak of heat release rate (pkHRR, $\left.\mathrm{kW} / \mathrm{m}^{2}\right)$, total heat release (THR, $\mathrm{MJ} / \mathrm{m}^{2}$ ), effective heat of combustion (EHC, MJ $/ \mathrm{kg}$ ), total smoke release (TSR, $\mathrm{m}^{2} / \mathrm{m}^{2}$ ) and post combustion residue were assessed. Each formulation investigated was tested four times in order to ensure reproducibility. The experimental error was calculated as standard deviation $(\sigma)$. Before being subjected to flammability and forced combustion tests, all the specimens were conditioned in a climatic chamber at $23 \pm 1{ }^{\circ} \mathrm{C}$ and $50 \%$ relative humidity for $48 \mathrm{~h}$.

\section{Results}

\subsection{Morphological and Chemical Characterization}

The morphology of the compacted $\mathrm{CH} / \mathrm{MMT}$ complexes after drying was investigated. Figure 2 reports digital images of the complexes before and after drying, FESEM observations of the film cross-section and the ATR spectrum of the film.
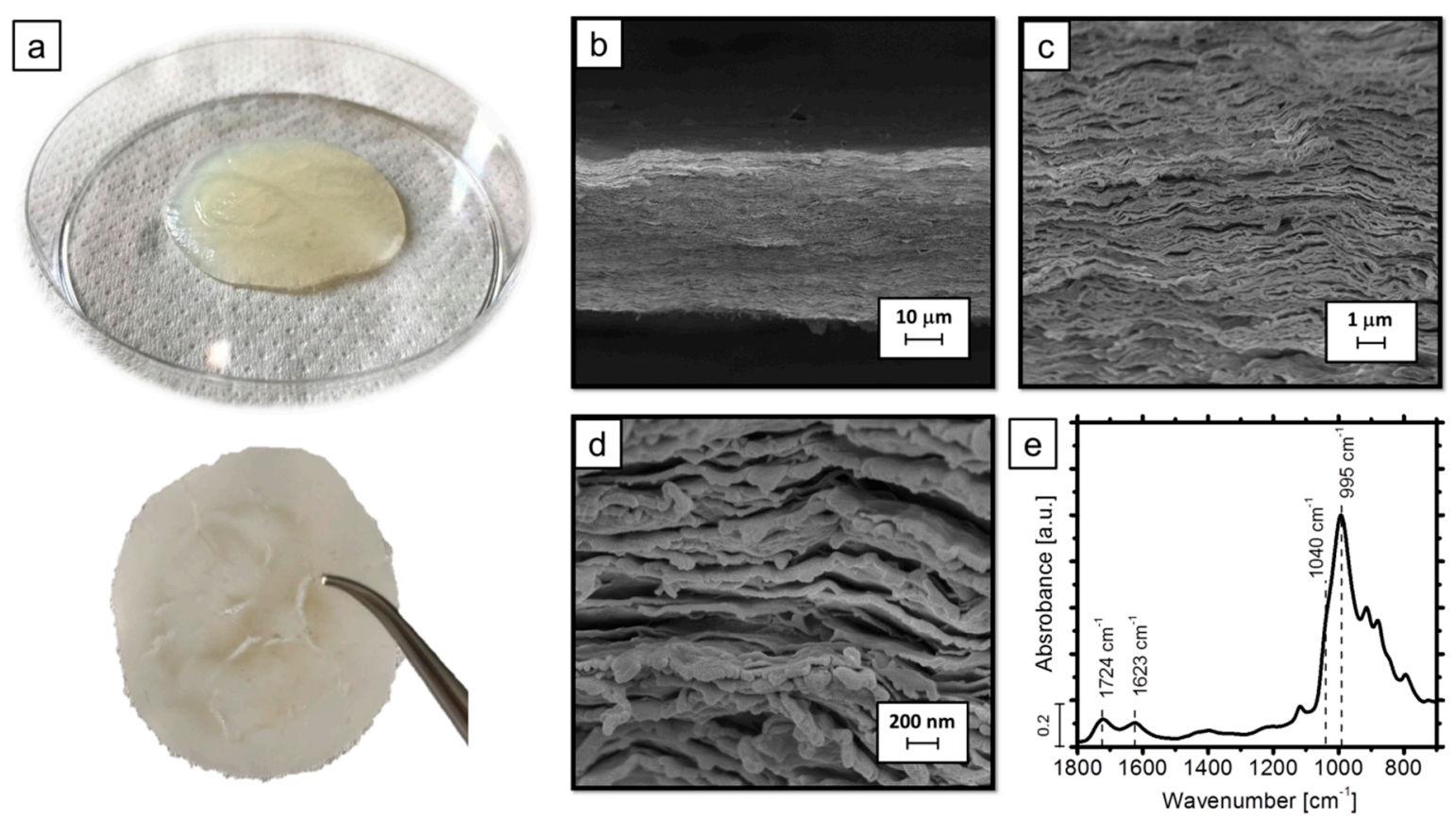

Figure 2. Digital images of the complexes before and after drying (a); field emission scanning electron microscopy (FESEM) observations of the film cross section at different magnifications (b-d) and attenuated total reflectance (ATR) spectrum of the film (e).

As reported in Figure 2a, upon drying the $\mathrm{CH}$ /MMT gel densified further, yielding a compact and thin film. FESEM micrographs of the film cross-section (Figure $2 \mathrm{~b}-\mathrm{d}$ ) revealed a nanoscale periodicity where the MMT platelets preferential orientation was apparent. The ATR spectrum of the dried film confirms the presence of both components by showing peaks ascribed to MMT and $\mathrm{CH}$ 
(Figure 2e). The former yields an intense peak at $995 \mathrm{~cm}^{-1}$ ascribed to Si-O-Si stretching vibration [31]. The latter yields minor signals related to $\mathrm{C}=\mathrm{O}$ stretching of citric acid at $1724 \mathrm{~cm}^{-1}$, a broad band centered at $1623 \mathrm{~cm}^{-1}$ due to N-H stretching of the protonated amine and $\mathrm{O}-\mathrm{H}$ bending and a shoulder at $1040 \mathrm{~cm}^{-1}$ ascribed to stretching vibrations of the C-O-C group in the glyosidic linkage [32]. These findings confirm the ability of the adopted procedure in depositing coatings characterized by multi-layered structures similar to those achievable by conventional LbL assembly.

In order to evaluate the changes in the surface morphology of acrylic fabrics after the treatment, the prepared samples were observed by SEM; Figure 3 reports the collected micrographs.
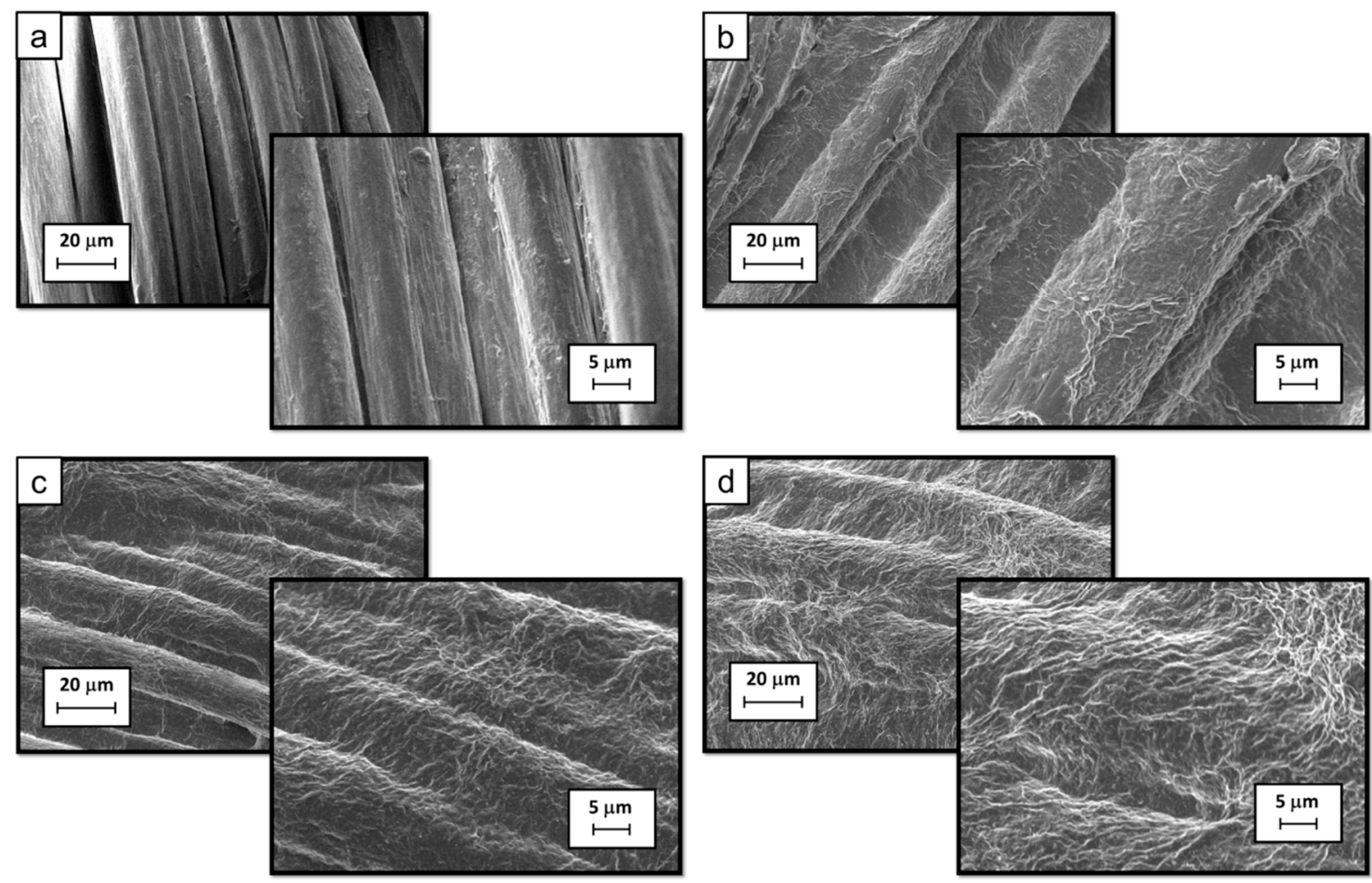

Figure 3. SEM micrographs of unmodified (a) and treated acrylic fabrics with $5 \%$ (b); $10 \%$ (c) and $20 \%$ (d) add-ons.

As reported in Figure 3a, neat acrylic fibers show a regular shape and a diameter with a surface morphology typical of wet spun fibers. The deposition of $\mathrm{CH} / \mathrm{MMT}$ complexes significantly changed the morphology of the fabric. Indeed, starting with the $5 \%$ add-on sample, it was possible to observe the presence of a continuous coating that covered the single fibers while joining them together, as clearly visible in Figure 3b. At 5\% add-on, the coating did not appear to be homogeneous in thickness, as high magnifications highlight the presence of untreated spots on the fiber surface. Increasing the add-on to 10 and $20 \%$, the increase of the surface coverage and homogeneity of the coating became more visible, as reported in Figure 3c,d. On the other hand, upon reaching the $20 \%$ add-on, it was possible to observe the formation of coating zones characterized by thicker aggregates (see inlet of Figure $3 \mathrm{~d}$ ). Untreated and treated fabrics were also analyzed by means of ATR-FTIR spectroscopy; the collected spectra are reported in Figure 4. 


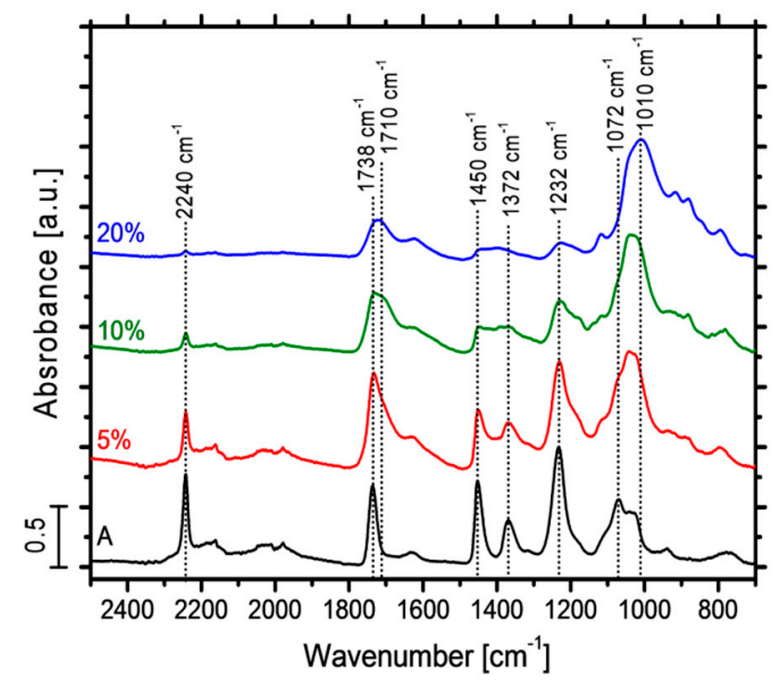

Figure 4. ATR spectra of untreated (A) and CH/MMT-treated acrylic fabrics with 5, 10 and $20 \%$ add-ons.

The spectrum of the acrylic fabric shows the typical peaks of a copolymer consisting of polyacrylonitrile and methyl acrylate. The peak at $2240 \mathrm{~cm}^{-1}$ can be ascribed to the $\mathrm{C} \equiv \mathrm{N}$ stretching of acrylonitrile unit while the peak at $1232 \mathrm{~cm}^{-1}$ can be related to $\mathrm{C}-\mathrm{N}$ stretching. C-H related signals can be found at $1450 \mathrm{~cm}^{-1}\left(\delta \mathrm{C}-\mathrm{H}\right.$ in $\left.\mathrm{CH}_{2}\right)$ and $1372 \mathrm{~cm}^{-1}(\delta \mathrm{C}-\mathrm{H}$ in $\mathrm{CH})$ while the strong peak at $1738 \mathrm{~cm}^{-1}$ is attributed to the ester group in comonomer used in the polymerization of the polyacrylonitrile $[33,34]$. The presence of the coating at different add-ons is responsible for an attenuation of the above-mentioned bands and the appearance of new peaks that grow in intensity as a function of the deposited add-on. The new signal found at $1710 \mathrm{~cm}^{-1}$ can be ascribed to the $\mathrm{C}=\mathrm{O}$ vibration of citric acid while the signal at $1010 \mathrm{~cm}^{-1}$ can be related to Si-O stretching vibration of MMT. As reported in Figure 4, such signals increase by increasing the coating add-on and at 20\% the Si-O-Si peak becomes the most intense.

\subsection{Flammability}

The reaction of untreated and treated fabrics to the application of a flame has been evaluated by horizontal flame spread tests. This test is of crucial importance as it mimics one of the most common scenarios that leads to the ignition and spread of a fire. Figure 5 reports some digital pictures of the fabrics during flammability tests and Table 1 summarizes the evaluated parameters.

Table 1. Flammability data of untreated and treated acrylic fabrics from horizontal flame spread tests.

\begin{tabular}{cccc}
\hline Sample & Flame Spread Rate $(\mathbf{m m} / \mathbf{s})$ & Dripping & Residue $(\mathbf{\%})$ \\
\hline A & $1.2 \pm 0.1$ & YES & $10 \pm 1$ \\
$5 \%$ & $1.0 \pm 0.2$ & NO & $20 \pm 2$ \\
$10 \%$ & $0.6 \pm 0.1$ & NO & $77 \pm 11$ \\
$20 \%$ & N.A. & NO & $93 \pm 3$ \\
\hline
\end{tabular}




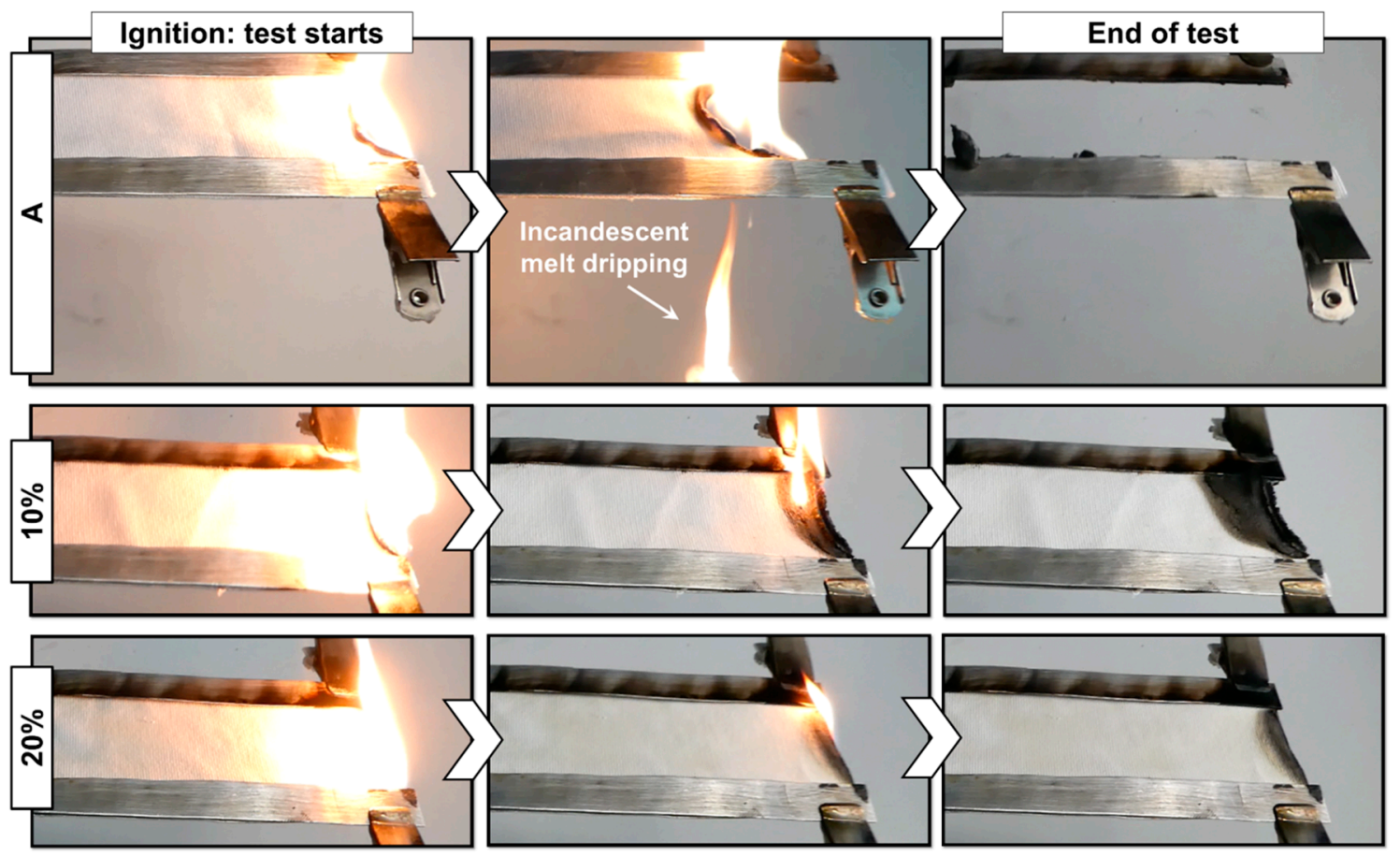

Figure 5. Digital pictures of flammability tests in horizontal configuration of untreated and treated acrylic fabrics. The first column reports the situation at few seconds after ignition, the second one during flame spread and third one after flame out.

Upon application of the methane flame the neat acrylic immediately caught fire and started burning with a vigorous flame that spread to the entire length of the sample. During combustion, the formation of incandescent droplets of flaming polymer ignited the dry cotton placed underneath the sample (central image in Figure 5). This behavior is typical of synthetic fibers and represents one of the most dangerous threats of these materials as this phenomenon can easily spread a fire to other ignitable materials close to the burning fabric (e.g., a carpet or moquette). At the end of the test, the only residue left was small portions of charred polymer attached to the metallic frame that account for an average $10 \%$ of the initial mass (see Figure 5).

The presence of the $\mathrm{CH} / \mathrm{MMT}$ coating completely changed the burning behavior of the samples. At $5 \%$ add-on, the formation of a coherent residue was observed; the presence of the coating slowed down the flame spread rate (Table 1) and prevented the incandescent melt-dripping phenomenon. Despite this, the flame still managed to spread to the entire length of the sample increasing the final residue to $20 \%$. When the coating add-on was increased to 10 or $20 \%$, the samples showed a self-extinguishing behavior, as they were able to stop the flame propagation and preserve a good portion of the fabric (see Figure 5). In detail, due to the presence of the coating, the advancing flame was confined to a progressively smaller portion of the sample where, due to the lack of combustible volatiles, it eventually extinguished. This occurred within the first $4.5 \mathrm{~cm}$ for the $10 \%$ samples, while for the $20 \%$ samples self-extinguishing occurred almost immediately after the removal of the methane flame. For this reason the final residues were as high as 77 and $93 \%$ for 10 and $20 \%$ add-on samples, respectively. The mentioned results were achieved thanks to the ordered brick and mortar structure and high clay loading of the deposited CH/MMT complexes. Indeed, this structure is well known to be capable of reducing both heat transmitted from the flame to the substrate and volatile release towards the flame $[16,30,35]$. This latter effect is expected to control the self-extinguishing behavior observed for 10 and $20 \%$ samples where the advancing flame is not capable of self-sustained combustion due to the lack of flammable volatiles. As clearly understandable from the performance of the treated fabrics, this FR action improved by increasing the coating thickness. 


\subsection{Forced Combustion}

Cone calorimetry tests were also performed in order to provide complementary information for the flammability evaluation. Untreated and treated fabrics were tested under a heat flux of $25 \mathrm{~kW} / \mathrm{m}^{2}$, which is typically found in the early stages of developing fires [36]. This heat flux was chosen as in the case of fire, fabrics normally ignite at low heat fluxes, contributing to fire development and spread. Figure 6 collects histograms of the most representative collected parameters, while Table 2 collects the complete set of data.
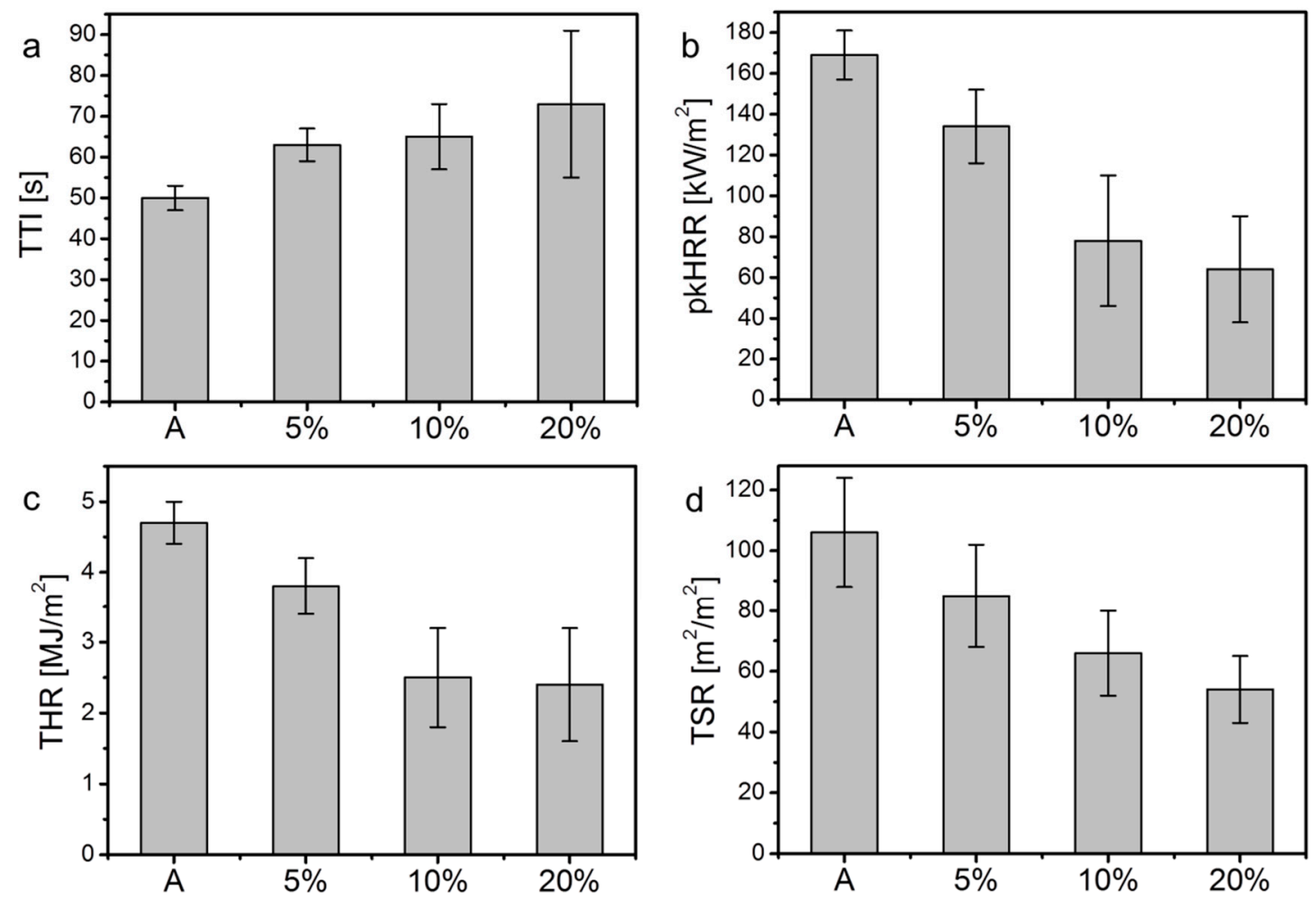

Figure 6. Histograms of the cone calorimetry data for untreated and treated fabrics: (a) time to ignition; (b) peak of heat release rate; (c) total heat release and (d) total smoke release.

Table 2. Cone calorimetry data of untreated and treated acrylic fabrics.

\begin{tabular}{|c|c|c|c|c|c|c|c|}
\hline Sample & $\underset{[\mathrm{s}]}{\mathrm{TTI} \pm \sigma}$ & $\begin{array}{c}\text { pkHRR } \pm \sigma \\
{\left[\mathrm{kW} / \mathrm{m}^{2}\right]} \\
\text { (Reduction, \%) }\end{array}$ & $\begin{array}{c}\text { FIGRA } \\
{\left[\mathrm{kW} / \mathrm{s} \mathrm{m}^{2}\right]}\end{array}$ & $\begin{array}{c}\text { THR } \pm \sigma \\
{\left[\mathrm{MJ} / \mathrm{m}^{2}\right]} \\
\text { (Reduction, \%) }\end{array}$ & $\begin{array}{c}\text { EHC } \pm \sigma \\
{[\mathrm{MJ} / \mathrm{kg}]} \\
\text { (Reduction, \%) }\end{array}$ & $\begin{array}{c}\text { TSR } \pm \sigma \\
{\left[\mathrm{m}^{2} / \mathrm{m}^{2}\right]} \\
\text { (Reduction, \%) }\end{array}$ & $\underset{[\%]}{\operatorname{Residue}} \pm \sigma$ \\
\hline $\mathrm{A}$ & $50 \pm 3$ & $169 \pm 12$ & $2.7 \pm 0.2$ & $4.7 \pm 0.3$ & $25.6 \pm 2.6$ & $106 \pm 18$ & 28 \\
\hline $5 \%$ & $63 \pm 4$ & $134 \pm 18(21)$ & $1.8 \pm 0.4$ & $3.8 \pm 0.4(20)$ & $22.1 \pm 2.8(13)$ & $85 \pm 17(19)$ & $42 \pm 4$ \\
\hline $10 \%$ & $65 \pm 8$ & $78 \pm 32(55)$ & $0.9 \pm 0.5$ & $2.5 \pm 0.7(48)$ & $18.0 \pm 5.0(30)$ & $66 \pm 14$ & $52 \pm 6$ \\
\hline $20 \%$ & $73 \pm 18$ & $64 \pm 26(62)$ & $0.7 \pm 0.3$ & $2.4 \pm 0.8(49)$ & $17.0 \pm 2.2(32)$ & $54 \pm 11(49)$ & $55 \pm 5$ \\
\hline
\end{tabular}

Upon being exposed to the cone heat flux, the neat acrylic first melted and then started decomposing with the release of flammable volatiles, which led to sample ignition after an average $50 \mathrm{~s}$. Then, the sample burnt with a vigorous flame, reaching a pkHRR of $169 \mathrm{~kW} / \mathrm{m}^{2}$. From an overall point of view the presence of the coating resulted in improvements proportional to the degree of coating add-on, for all the observed parameters. Indeed, all treated fabrics showed an increase in TTI and a reduction in the heat-release-related parameters (see pkHRR, THR and EHC values in Table 2); in addition, substantial reductions in the TSR values (up to $49 \%$ for $20 \%$ samples) were also observed. The best results were achieved by the $20 \%$ samples, which postponed ignition by $23 \mathrm{~s}$ and reduced the pkHRR and THR by 62 and $49 \%$, respectively. As already observed from flammability tests, thanks 
to the achieved brick and mortar structure, the deposited coating efficiently controlled the heat and most importantly mass transfer before and after ignition. Before ignition, the reduced heat transfer and limited volatile release resulted in prolonged TTI values. On the other hand, during combustion, the coating still limited the rate at which volatiles were released and in this manner strongly affected the HRR values. This combined protection also promoted the char formation from acrylic fibers, as pointed out by the increase in final residue values and the related decrease in THR, as less volatiles are burned during combustion. Further proof of this condensed phase volatile barrier effect can be found by observing a substantial reduction (up to $32 \%$ for $20 \%$ samples) in EHC values. In order to provide an overall evaluation of the FR performance achieved by the treated fabrics, the fire growth rate (FIGRA) values were calculated. This is a fire safety parameter obtained by the ratio between the pkHRR and the time at which the peak occurs; high FIGRA values are normally associated with rapid fire growth and spread. As reported in Table 2, due to the increase of TTI and the reduction of pkHRR, all treated fabrics managed to lower this parameter, further demonstrating the high FR efficiency of the deposited coatings.

\section{Conclusions}

The present work investigated the use of chitosan/montmorillonite complexes obtained by the direct mixing of an oppositely charged solution/suspension for the deposition of flame retardant coatings on acrylic fibers. The compaction procedure using centrifugal forces resulted our obtaining a pearlescent gel, the viscosity of which allowed it to be used in doctor-blading coatings. The structure of the dried complexes was investigated by FESEM observation revealing a multi-layered structure, typical of LbL assembled coatings, in which oriented clay nanoplatelets are held together by a continuous chitosan matrix. When deposited on the surface of acrylic fabrics, this $\mathrm{CH} / \mathrm{MMT}$ complex resulted in a continuous coating that covers the surface of the fibers, while also linking them together. A total of 5\% add-on yielded uncoated spots and non-homogeneous thicknesses. Increasing the coating add-on to 10 or $20 \%$ in weight improved the homogeneity of the surface coverage. From the flammability point of view, all treated fabrics were capable of suppressing the melt-dripping phenomenon that was observed for the neat fabrics. The 10 and 20\% add-ons achieved self-extinguishing behavior by stopping flame propagation and preserving good portions of the fabrics. The best performance was achieved by the $20 \%$ add-on sample, which self-extinguished the flame within a few seconds of initial flame application. During forced combustion tests, the presence of the coating increased the time required to achieve ignition and considerably reduced the rates at which heat was released, thus positively affecting HRR, THR and EHC values. A reduction of TSR was also observed. The good flame retardancy displayed by the treated fabrics was associated with the heat and volatility barrier exerted by the deposited $\mathrm{CH} / \mathrm{MMT}$ complexes, which eventually also improved the char-forming ability of the acrylic fibers. In particular, the reduction/hindrance of flammable volatile release was identified as one of the key factors for the achievement of self-extinguishing behavior in flammability tests, as well as considerably reducing the heat release and smoke release rates during forced combustion tests. In conclusion, the proposed approach allows for the efficient deposition of coatings characterized by LbL-like structure and interactions with promising FR retardant properties. This was achieved by the use of renewable materials and exploiting a simple and fast water-based process. Further developments might be directed towards the improvement of fabric hand and coating durability.

Author Contributions: Conceptualization, F.C. and J.A.; Investigation, F.C.; Writing-original draft, F.C.; Writing-review and editing, J.A.

Acknowledgments: The authors want to thank Mr. Fabio Cuttica for the cone calorimetry tests and Mr. Mauro Raimondo for the FESEM observations.

Conflicts of Interest: The authors declare no conflict of interest. 


\section{References}

1. Sen, K.; Bahrami, S.H.; Bajaj, P. High-performance acrylic fibers. J. Macromol. Sci. Part C Polym. Rev. 1996, 36, 1-76. [CrossRef]

2. Alongi, J.; Ra, H.; Carosio, F.; Malucelli, G. Update on Flame Retardant Textiles: State of the Art, Environmental Issues and Innovative Solutions; Smithers Rapra Technology Ltd.: Shawbury, UK, 2013.

3. Bajaj, P.; Kumari, S. Modification of acrylic fibers: An overview. J. Macromol. Sci. Rev. Macromol. Chem. Phys. 1987, 27, 181-217. [CrossRef]

4. Tsai, J.-S. The effect of flame-retardants on the properties of acrylic and modacrylic fibres. J. Mater. Sci. 1993, 28, 1161-1167. [CrossRef]

5. Hall, M.E.; Zhang, J.; Richard Horrocks, A. The flammability of polyacrylonitrile and its copolymers III. Effect of flame retardants. Fire Mater. 1994, 18, 231-241. [CrossRef]

6. Hall, M.E.; Horrocks, A.R.; Zhang, J. The flammability of polyacrylonitrile and its copolymers. Polym. Degrad. Stabil. 1994, 44, 379-386. [CrossRef]

7. Chou, S.; $\mathrm{Wu}, \mathrm{C}$.-J. Effect of brominated flame retardants on the properties of acrylonitrile/vinyl acetate copolymer fibers. Text. Res. J. 1995, 65, 533-539. [CrossRef]

8. Malucelli, G.; Carosio, F.; Alongi, J.; Fina, A.; Frache, A.; Camino, G. Materials engineering for surface-confined flame retardancy. Mater. Sci. Eng. R 2014, 84, 1-20. [CrossRef]

9. Alongi, J.; Carosio, F.; Kiekens, P. Recent advances in the design of water based-flame retardant coatings for polyester and polyester-cotton blends. Polymers 2016, 8, 357. [CrossRef]

10. Holder, K.M.; Smith, R.J.; Grunlan, J.C. A review of flame retardant nanocoatings prepared using layer-by-layer assembly of polyelectrolytes. J. Mater. Sci. 2017, 52, 12923-12959. [CrossRef]

11. Decher, G. Fuzzy nanoassemblies: Toward layered polymeric multicomposites. Science 1997, 277, $1232-1237$. [CrossRef]

12. Kabanov, V.A. Multilayer Thin Films: Sequential Assembly of Nanocomposite Materials, 2nd ed.; Wiley-VCH: Weinheim, Germany, 2012; p. 1112.

13. Patra, D.; Vangal, P.; Cain, A.A.; Cho, C.; Regev, O.; Grunlan, J.C. Inorganic nanoparticle thin film that suppresses flammability of polyurethane with only a single electrostatically-assembled bilayer. ACS Appl. Mater. Interfaces 2014, 6, 16903-16908. [CrossRef] [PubMed]

14. Carosio, F.; Di Blasio, A.; Alongi, J.; Malucelli, G. Layer by layer nanoarchitectures for the surface protection of polycarbonate. Eur. Polym. J. 2013, 49,397-404. [CrossRef]

15. Cain, A.A.; Plummer, M.G.B.; Murray, S.E.; Bolling, L.; Regev, O.; Grunlan, J.C. Iron-containing, high aspect ratio clay as nanoarmor that imparts substantial thermal/flame protection to polyurethane with a single electrostatically-deposited bilayer. J. Mater. Chem. A 2014, 2, 17609-17617. [CrossRef]

16. Laufer, G.; Kirkland, C.; Cain, A.A.; Grunlan, J.C. Clay-chitosan nanobrick walls: Completely renewable gas barrier and flame-retardant nanocoatings. ACS Appl. Mater. Interfaces 2012, 4, 1643-1649. [CrossRef] [PubMed]

17. Carosio, F.; Fontaine, G.; Alongi, J.; Bourbigot, S. Starch-based layer by layer assembly: Efficient and sustainable approach to cotton fire protection. ACS Appl. Mater. Interfaces 2015, 7, 12158-12167. [CrossRef] [PubMed]

18. Carosio, F.; Negrell-Guirao, C.; Alongi, J.; David, G.; Camino, G. All-polymer layer by layer coating as efficient solution to polyurethane foam flame retardancy. Eur. Polym. J. 2015, 70, 94-103. [CrossRef]

19. Koklukaya, O.; Carosio, F.; Wãgberg, L. Superior flame-resistant cellulose nanofibril aerogels modified with hybrid layer-by-layer coatings. ACS Appl. Mater. Interfaces 2017, 9, 29082-29092. [CrossRef] [PubMed]

20. Guin, T.; Krecker, M.; Milhorn, A.; Grunlan, J.C. Maintaining hand and improving fire resistance of cotton fabric through ultrasonication rinsing of multilayer nanocoating. Cellulose 2014, 21, 3023-3030. [CrossRef]

21. Carosio, F.; Negrell-Guirao, C.; Di Blasio, A.; Alongi, J.; David, G.; Camino, G. Tunable thermal and flame response of phosphonated oligoallylamines layer by layer assemblies on cotton. Carbohydr. Polym. 2015, 115, 752-759. [CrossRef] [PubMed]

22. Carosio, F.; Di Pierro, A.; Alongi, J.; Fina, A.; Saracco, G. Controlling the melt dripping of polyester fabrics by tuning the ionic strength of polyhedral oligomeric silsesquioxane and sodium montmorillonite coatings assembled through layer by layer. J. Colloid Interface Sci. 2018, 510, 142-151. [CrossRef] [PubMed] 
23. van der Gucht, J.; Spruijt, E.; Lemmers, M.; Stuart, M.A.C. Polyelectrolyte complexes: Bulk phases and colloidal systems. J. Colloid Interface Sci. 2011, 361, 407-422. [CrossRef] [PubMed]

24. Negrell-Guirao, C.; Carosio, F.; Boutevin, B.; Cottet, H.; Loubat, C. Phosphonated oligoallylamine: Synthesis, characterization in water, and development of layer by layer assembly. J. Polym. Sci. Pol. Phys. 2013, 51, 1244-1251. [CrossRef]

25. Ghanadpour, M.; Carosio, F.; Wågberg, L. Ultrastrong and flame-resistant freestanding films from nanocelluloses, self-assembled using a layer-by-layer approach. Appl. Mater. Today 2017, 9, 229-239. [CrossRef]

26. Cain, A.A.; Murray, S.; Holder, K.M.; Nolen, C.R.; Grunlan, J.C. Intumescent nanocoating extinguishes flame on fabric using aqueous polyelectrolyte complex deposited in single step. Macromol. Mater. Eng. 2014, 299, 1180-1187. [CrossRef]

27. Haile, M.; Fincher, C.; Fomete, S.; Grunlan, J.C. Water-soluble polyelectrolyte complexes that extinguish fire on cotton fabric when deposited as ph-cured nanocoating. Polym. Degrad. Stabil. 2015, 114, 60-64. [CrossRef]

28. Schaaf, P.; Schlenoff, J.B. Saloplastics: Processing compact polyelectrolyte complexes. Adv. Mater. 2015, 27, 2420-2432. [CrossRef] [PubMed]

29. Haile, M.; Sarwar, O.; Henderson, R.; Smith, R.; Grunlan, J.C. Polyelectrolyte coacervates deposited as high gas barrier thin films. Macromol. Rapid Commun. 2017, 38. [CrossRef] [PubMed]

30. Carosio, F.; Kochumalayil, J.; Cuttica, F.; Camino, G.; Berglund, L. Oriented clay nanopaper from biobased components-mechanisms for superior fire protection properties. ACS Appl. Mater. Interfaces 2015, 7, 5847-5856. [CrossRef] [PubMed]

31. Socrates, G. Infrared and Raman Characteristic Group Frequencies-Table and Charts, 3rd ed.; Wiley: Weinheim, Germany, 2006.

32. Silva, S.M.; Braga, C.R.; Fook, M.V.; Raposo, C.M.; Carvalho, L.H.; Canedo, E.L. Application of infrared spectroscopy to analysis of chitosan/clay nanocomposites, infrared spectroscopy. In Infrared Spectroscopy-Materials Science, Engineering and Technology; InTech: London, UK, 2012.

33. Dalton, S.; Heatley, F.; Budd, P.M. Thermal stabilization of polyacrylonitrile fibres. Polymer 1999, 40, 5531-5543. [CrossRef]

34. Carosio, F.; Alongi, J. Influence of layer by layer coatings containing octapropylammonium polyhedral oligomeric silsesquioxane and ammonium polyphosphate on the thermal stability and flammability of acrylic fabrics. J. Anal. Appl. Pyrol. 2016, 119, 114-123. [CrossRef]

35. Carosio, F.; Cuttica, F.; Medina, L.; Berglund, L.A. Clay nanopaper as multifunctional brick and mortar fire protection coating-Wood case study. Mater. Des. 2016, 93, 357-363. [CrossRef]

36. Schartel, B.; Hull, T.R. Development of fire-retarded materials-Interpretation of cone calorimeter data. Fire Mater. 2007, 31, 327-354. [CrossRef]

(C) 2018 by the authors. Licensee MDPI, Basel, Switzerland. This article is an open access article distributed under the terms and conditions of the Creative Commons Attribution (CC BY) license (http://creativecommons.org/licenses/by/4.0/). 